\title{
Multiple-Mode Diffusion Waves for Viscous Nonstrictly Hyperbolic Conservation Laws *
}

\section{I-Liang Chern}

Mathematics and Computer Science Division, Argonne National Laboratory, Argonne, IL 60439-4801, USA and

Department of Mathematics, The University of Chicago, Chicago, IL 60637, USA

Received February 13, 1990; in revised form November 13, 1990

\begin{abstract}
We study the large-time behaviors of solutions of viscous conservation laws whose inviscid part is a nonstrictly hyperbolic system. The initial data considered here is a perturbation of a constant state. It is shown that the solutions converge to single-mode diffusion waves in directions of strictly hyperbolic fields, and to multiple-mode diffusion waves in directions of nonstrictly hyperbolic fields. The multiple-mode diffusion waves, which are the new elements here, are the selfsimilar solutions of the viscous conservation laws projected to the nonstrictly hyperbolic fields, with the nonlinear fluxes replaced by their quadratic parts. The convergence rate to these diffusion waves is $O\left(t^{-3 / 4+1 / 2 p+\sigma}\right)$ in $L^{p}, 1 \leqq p \leqq \infty$, with $\sigma>0$ being arbitrarily small.
\end{abstract}

\section{Introduction}

We are interested in the large-time behaviors of solutions of the viscous conservation laws

$$
u_{t}+f(u)_{x}=u_{x x}, \quad u \in R^{n},-\infty<x<\infty, t>0,
$$

whose inviscid part is a nonstrictly hyperbolic system. Physical models of the nonstrictly hyperbolic systems include, for instance, three-phase flows in oil reservoir [10]. The initial data considered here is a perturbation of a constant state. Without loss of generality, we may assume this constant state to be the zero state

$$
u(x, 0)=u_{0}(x), u_{0}(x) \rightarrow 0, \quad \text { as } \quad|x| \rightarrow \infty .
$$

The viscous term $u_{x x}$ considered here is an idealized situation. The real physics has a more general viscous term $\left(B(u) u_{x}\right)_{x}$, where $B$ is an $n \times n$ matrix. Kawashima showed that the nondiagonal part of $\left(B(u) u_{x}\right)_{x}$ decays faster than the diagonal

* This work was supported by the Applied Mathematical Sciences subprogram of the Office of Energy Research, U.S. Department of Energy, under Contract W-31-109-Eng-38 
part in the $L^{2}$ sense [5]. Therefore, the idealized model (1.1) can still capture the essential large-time phenomena.

When the inviscid part of (1.1) is strictly hyperbolic, the following results have been shown. At the large time, the solutions of (1.1) and (1.2) decay in $L^{p}$, for all $1<p \leqq \infty$, because the viscous terms dominate the nonlinear terms if the perturbation is small (see [4] and [9] and the references therein). The solutions do not decay in $L^{1}$ because of the conservation of masses of (1.1):

$$
\int u(x, t) d x=\text { const . }
$$

In the $L^{1}$ behaviors of the solutions of (1.1) and (1.2), the hyperbolic terms play an important role. The strict hyperbolicity causes the systems to decouple at the large time. As a consequence, the $L^{1}$ asymptote of (1.1) and (1.2) consists of so-called diffusion waves in each characteristic direction [1]. These diffusion waves carry the invariant masses of the solutions and are the self-similar solutions of the Burgers equations. The rate of convergence to these diffusion waves is $O\left(t^{-1 / 4+\sigma}\right)$ in $L^{1}$ with $\sigma>0$ being arbitrarily small [1]. The optimal rate occurs when $\sigma=0$ [2]. Independently, Kawashima also showed an $L^{2}$ result for more general hyperbolicparabolic systems [5]. The above $L^{1}$ result is consistent with the inviscid result of Liu [7], where the rate of convergence to the $N$-waves is $O\left(t^{-1 / 4}\right)$ in $L^{1}$. However, it is not known whether this is the optimal rate.

In this paper, we investigate the cases when the inviscid part of (1.1) is nonstrictly hyperbolic. Two different situations can arise - the nonresonant and resonant - depending on whether $f^{\prime}(0)$ is diagonalizable or not. In the latter case, Liu and Xin [8] recently showed that the $L^{1}$ norms of the solutions of Temple's equation [11] (an example of the resonant case) tend to $\infty$ as $t \rightarrow \infty$. There, the nontrivial Jordan form of $f^{\prime}(0)$ is responsible for this resonancy. Thus, the linear resonancy is the dominant phenomenon.

Here, we study the formal case, the nonresonant case. We further assume that $f^{\prime \prime}(0)$ exists. A physical model belonging to this case is the complex Burgers equation near the umbilical point, which models the three-phase flow of an oil reservoir [10]. In this nonresonant case, the large-time behaviors of the solutions are more like those of the strictly hyperbolic viscous systems; specifically, the solutions do not blow up in $L^{1}$ and their $L^{1}$ asymptotic behaviors are nonlinear. The difference is that, in the current situation, the fields having the same characteristic speeds do not decouple at the large time. As a result, multiple-mode diffusion waves form in these directions. These multiple-mode diffusion waves share two important properties of the diffusion waves of the strictly hyperbolic viscous systems, namely, carrying constant masses and decaying as heat kernels.

Our basic assumption is that the inviscid part of (1.1)

$$
u_{t}+f(u)_{x}=0
$$

is nonstrictly hyperbolic at $u=0$. This means that $f^{\prime}(0)$ has a complete set of real right eigenvectors $r_{1}, \ldots, r_{n}$, and the associated eigenvalues $\lambda_{1}, \ldots, \lambda_{n}$ are all real but may coincide. We further assume that $f^{\prime}(0)$ is diagonalizable and $f^{\prime \prime}(0)$ exists. For simplicity of notation and without loss of generality, we may assume

$$
\begin{gathered}
\lambda_{1}=\ldots=\lambda_{r}<\lambda_{r+1}<\ldots<\lambda_{n}, \\
f^{\prime}(0)=\left(\begin{array}{lll}
\lambda_{1} & & 0 \\
& \ddots & \\
0 & & \lambda_{n}
\end{array}\right),
\end{gathered}
$$


where the right eigenvectors $r_{i}=\left(\delta_{i 1}, \ldots, \delta_{i n}\right)^{t}$ and the left eigenvectors $l_{i}=\left(\delta_{i 1}, \ldots, \delta_{i n}\right)$. Below, we define the single-mode and the multiple-mode diffusion waves for (1.1) and (1.2). They carry the invariant masses

$$
m_{i} \equiv l_{i} \cdot \int u(x, t) d x, \quad i=1, \ldots, n .
$$

For each strictly hyperbolic fields $i=r+1, \ldots, n$, the single-mode diffusion wave is defined to be $\theta_{i}(x, t) r_{i}$, where $\theta_{i}$ is the self-similar solution of the Burgers equation

$$
\theta_{i t}+\lambda_{i} \theta_{i x}+\frac{1}{2} b_{i i i}\left(\theta_{i}^{2}\right)_{x}=\theta_{i x x}
$$

carrying the mass

$$
\int \theta_{i}(x, t) d x=m_{i} .
$$

Here, $b_{i i i}=l_{i} \cdot\left(f^{\prime \prime}(0) r_{i}, r_{i}\right) \cdot \theta_{i}$ has the following explicit expression [3]:

$$
\begin{gathered}
\theta_{i}(x, t)=\frac{1}{\sqrt{t}} \theta_{i}^{*}\left(\frac{x-\lambda_{i} t}{\sqrt{t}}\right), \\
\theta_{i}^{*}(\xi)=\left\{\begin{array}{cc}
\frac{m_{i}}{\sqrt{4 \pi}} e^{-\xi^{2} / 4} & \text { if } \quad b_{i i i}=0 \\
-\frac{1}{b_{i i i}}\left(\ln \psi_{i}(\xi)\right)^{\prime} & \text { if } \quad b_{i i i} \neq 0
\end{array}\right. \\
\psi_{i}(\xi)=e^{b_{i i i} m_{i} / 4} \int_{-\infty}^{\xi} e^{-y^{2} / 4} d y+e^{-b_{i i i} m_{i} / 4} \int_{\xi}^{\infty} e^{-y^{2} / 4} d y .
\end{gathered}
$$

In the directions of the nonstrictly hyperbolic fields (i.e., $i=1, \ldots, r$ ), the invariant mass vector $m \equiv \sum_{1}^{r} m_{i} r_{i}$ is carried by the multiple-mode diffusion wave $\theta=\sum_{i=1}^{r} \theta_{i} r_{i}$, which is defined to be the self-similar solution of the generalized Burgers equation in $R^{r}$

$$
\begin{gathered}
\theta_{t}+\lambda \theta_{x}+\frac{1}{2} Q(\theta)_{x}=\theta_{x x}, \\
\lambda \equiv \lambda_{1}, \quad Q(\theta)=\left(\begin{array}{c}
l_{1} \\
\vdots \\
l_{r}
\end{array}\right) \cdot\left(f^{\prime \prime}(0) \theta, \theta\right),
\end{gathered}
$$

carrying the mass vector

$$
\int \theta(x, t) d x=m .
$$

Theorem 1. For small $m \in R^{r},(1.8),(1.9)$ has a unique self-similar solution of the form $\theta(x, t)=\frac{1}{\sqrt{t}} \phi\left(\frac{x-\lambda t}{\sqrt{t}}\right)$, with $\phi(-\infty)=\phi^{\prime}(-\infty)=0$. Furthermore, $\phi$ has the property

$$
\phi(\xi)=e^{-\xi^{2} / 4} \psi(\xi)
$$

where $\psi$ and all its derivatives are uniformly bounded.

The main result of this paper is to show that the solution $u(x, t)$ of (1.1) and (1.2) converges to the above diffusion waves. The assumption on the initial data $u_{0}(x)$ is 
that $u_{0} \in L_{\beta}^{1}$ for some $\beta>0$, where

$$
\left\|u_{0}\right\|_{L_{\beta}^{1}} \equiv \int(1+|x|)^{\beta}\left|u_{0}(x)\right| d x .
$$

The solution $u$ we are seeking is a weak solution in the class $C\left((0, \infty), L^{1} \cap L^{\infty}\right)$.

Theorem 2. Consider the initial value problem (1.1) and (1.2). If we assume that $\left\|u_{0}\right\|_{L_{\beta}^{1} \beta}$ is sufficiently small for some $\beta>0$, then there exists a unique solution $u(x, t)$ of (1.1), (1.2) in $C\left((0, \infty), L^{1} \cap L^{\infty}\right)$. Moreover, $u$ is indeed in $C^{\infty}((0, \infty) \times R)$. Furthermore, let the single-mode diffusion waves $\theta_{i} r_{i}, i=r+1, \ldots, n$, be defined by (1.5) and (1.6) and the multiple-mode diffusion waves $\sum_{1}^{r} \theta_{i} r_{i}$ be defined by (1.8) and (1.9).
Then

$$
\left\|u(\cdot, t)-\sum_{i=1}^{n} \theta_{i} r_{i}(\cdot, t)\right\|_{L^{p}}=O\left(t^{-1 / 2+1 / 2 p-\bar{\beta} / 2+\sigma}\right), \text { as } t \rightarrow \infty,
$$

where $\bar{\beta}=\min \{\beta, 1 / 2\}$ and $\sigma$ is an arbitrarily small constant.

The basic idea of the proof of this main theorem is slightly different from that in $[1,6]$, namely, we perform $L^{1}$ and $L^{\infty}$ estimations instead of $L^{2}$ estimations performed in $[1,6]$. This simplifies the proof. Another new element here is the estimation of the convection of the transversal multiple-mode diffusion waves. This was not considered in the construction of the "hyperbolic waves" introduced by Liu [5]. The estimation here not only can treat the multiple-mode diffusion waves, but also greatly simplifies Liu's estimation for the hyperbolic waves [5]. This estimation will be discussed in detail in the last section; the remainder of the proof of this theorem will only be sketched in this paper.

\section{The Multiple-Mode Diffusion Waves}

In this section, we prove Theorem 1. Instead of considering Eq. (1.8) with a uniform viscous term, we consider the following general viscous system:

$$
A_{r}^{0} \theta_{t}+\lambda A_{r}^{0} \theta_{x}+\frac{1}{2} Q(\theta)_{x}=B_{r} \theta_{x x} .
$$

Here, $A_{r}^{0}$ and $B_{r}$ are symmetric $r \times r$ matrices, and $A_{r}^{0}$ is positive definite. We assume the following stability condition: All eigenvalues of $B_{r}$ with respect to $A_{r}^{0}$ satisfy

$$
\alpha_{i}>0, \quad i=1, \ldots, r .
$$

This assumption is satisfied for those symmetrizable hyperbolic-parabolic systems considered by Kawashima $[4,5]$, which include many interesting physical models such as the compressible Navier-Stokes equation.

Theorem 3. For small $m \in R^{r},(2.1)$ and (1.9) have a unique self-similar solution of the form $\theta(x, t)=\frac{1}{\sqrt{t}} \phi\left(\frac{x-\lambda t}{\sqrt{t}}\right)$, with $\phi(-\infty)=\phi^{\prime}(-\infty)=0$. Furthermore, $\phi$ has the following property:

$$
\begin{gathered}
\phi(\xi)=e^{-\xi^{2} / 4 \alpha} \psi(\xi), \\
\alpha=\max \left\{\alpha_{1}, \ldots, \alpha_{r}\right\},
\end{gathered}
$$

where $\psi$ and all its derivatives are uniformly bounded. 
Proof. Substituting $\theta(x, t)=\frac{1}{\sqrt{t}} \phi\left(\frac{x-\lambda t}{\sqrt{t}}\right)$ in (2.1), we obtain

$$
-\frac{1}{2} A_{r}^{0}(\xi \phi)^{\prime}+\frac{1}{2} Q(\phi)^{\prime}=B_{r} \phi^{\prime \prime} .
$$

Integrating this equation and using the assumption $\phi(-\infty)=\phi^{\prime}(-\infty)=0$, we obtain

$$
B_{r} \phi^{\prime}=-\frac{1}{2} \xi A_{r}^{0} \phi+\frac{1}{2} Q(\phi) .
$$

Let $\psi(\xi) \equiv e^{\xi^{2} / 4 \alpha} \phi(\xi)$, and let $p_{i}$ and $q_{i}, i=1, \ldots, r$ be the right and left eigenvectors of $B_{r}$ with respect to $A_{r}^{0}$. Expanding $\psi=\sum_{i=1}^{r} \psi_{i} p_{i}$ and using the fact that $Q$ is a quadratic function, we find that $\psi_{i}$ satisfies

$$
\psi_{i}^{\prime}(\xi)=-\frac{\xi}{2}\left(\frac{1}{\alpha_{i}}-\frac{1}{\alpha}\right) \psi_{i}(\xi)+e^{-\xi^{2} / 4 \alpha} Q_{i}(\psi), \quad i=1, \ldots, r,
$$

where $Q_{i}=\frac{1}{2 \alpha_{i}} q_{i} A_{r}^{0} Q$. Next, we write these equations in integral forms. With the abbreviation $\frac{1}{4}\left(1 / \alpha_{i}-1 / \alpha\right)=\mu_{i}$, the function $\psi$ satisfies the following integral equation:

$$
\begin{aligned}
\psi(\xi) & =\sum_{i=1}^{r}\left(e^{-\mu_{i} \xi^{2}} \psi_{i}(0)+\int_{0}^{\xi} e^{-\mu_{i}\left(\xi^{2}-\eta^{2}\right)} e^{-\eta^{2} / 4 \alpha} Q_{i}(\psi(\eta)) d \eta\right) p_{i} \\
& \equiv T(\psi)(\xi) .
\end{aligned}
$$

From (2.4) and the integrability of $e^{-\eta^{2} / 4 \alpha}$ over $R$, we see that $T$ is an operator from $C_{b}\left(\right.$ all $R^{r}$-valued bounded continuous functions on $R$ with the sup norm $\left.\|\cdot\|_{\infty}\right)$ to itself and

$$
\begin{gathered}
\|T(\psi)\|_{\infty} \leqq|\psi(0)|+C \sqrt{\alpha \pi}\|\psi\|_{\infty}^{2}, \\
\left\|T\left(\psi_{1}\right)-T\left(\psi_{2}\right)\right\|_{\infty} \leqq C \sqrt{\alpha \pi}\left(\left\|\psi_{1}\right\|_{\infty}^{2}-\left\|\psi_{2}\right\|_{\infty}^{2}\right) .
\end{gathered}
$$

Here, $C$ is a constant bounding $\left|A_{r}^{0} Q(\psi)\right| /|\psi|^{2}$ from above. Thus, the existence and the uniqueness of solution of (2.8) in $C_{b}$ follow from the standard fixed-point theorem for contraction mappings, which yields

provided

$$
\|\psi\|_{\infty}<\frac{1}{2 C \sqrt{\alpha \pi}},
$$

$$
|\psi(0)|<\frac{1}{4 C \sqrt{\alpha \pi}} .
$$

The uniform boundedness of higher-order derivatives of $\psi$ follows from (2.7).

Notice that the masses carried by the self-similar solution obtained above are always invariant in time:

$$
\int \frac{1}{\sqrt{t}} \phi\left(\frac{x-\lambda t}{\sqrt{t}}\right) d x=\int \phi(\xi) d \xi,
$$

and the derivative of the mapping

$$
\phi(0) \mapsto \int \phi(\xi) d \xi
$$


at $\phi(0)=0$ is equivalent to

$$
2 \sqrt{\pi}\left(\begin{array}{ccc}
\sqrt{\alpha_{1}} & & 0 \\
& \ddots & \\
0 & & \sqrt{\alpha_{r}}
\end{array}\right),
$$

which is nonsingular. Thus, the inverse function theorem yields that, given $m \in R^{r}$ sufficiently small, there exists a unique (the above) self-similar solution that carries the mass $m$.

Notice that the expression (2.3) implies that the multiple-mode diffusion waves decay as heat kernels: for $1 \leqq p \leqq \infty, l \geqq 0$,

$$
\begin{gathered}
\left\|D_{x}^{l} \theta(\cdot, t)\right\|_{L^{p}}=O\left(t^{-l / 2-1 / 2+1 / 2 p}\right), \\
\left\|D_{t}^{l} \theta(\cdot, t)\right\|_{L^{p}}=O\left(t^{-l-1 / 2+1 / 2 p}\right) .
\end{gathered}
$$

\section{Proof of Theorem 2}

This section is devoted to the proof of Theorem 2. First, we may replace $\theta_{i}\left(\frac{x-\lambda_{i} t}{\sqrt{t}}\right)$ by $\theta_{i}\left(\frac{x-\lambda_{i}(t+1)}{\sqrt{t+1}}\right)$ to avoid the singularity of the diffusion waves at $t=0$. This change does not affect the convergent rate, for

$$
\left\|D_{x}^{l}\left(\theta_{i}\left(\frac{x-\lambda_{i}(t+1)}{\sqrt{t+1}}\right)-\theta_{i}\left(\frac{x-\lambda_{i} t}{\sqrt{t}}\right)\right)\right\|_{L^{p}}=O\left(t^{-l / 2-3 / 2+1 / 2 p}\right),
$$

which follows from (2.13) and (2.14). Next, we let

$$
w(x, t)=u(x, t)-\sum_{i=1}^{n} \theta_{i}\left(\frac{x-\lambda_{i}(t+1)}{\sqrt{t+1}}\right) r_{i} .
$$

The proof of Theorem 2 is composed of (i) a local existence and regularity theorem for $w$, (ii) a local uniqueness theorem for $w$, and (iii) an a priori decay estimate for $w$. (i) and (ii) are standard; see, for instance, [5, Theorem 2.9]. The a priori estimate we are seeking is described as follows. For $0<\beta \leqq 1$, for any arbitrarily small positive number $\sigma$ and for any fixed $T>0$, define

$$
\begin{aligned}
\|w\|_{T} \equiv & \sup _{0<t \leqq T}(1+t)^{\bar{\beta}-\sigma}\|w(\cdot, t)\|_{L^{1}} \\
& +\sup _{0<t \leqq T}(1+t)^{1 / 2+\bar{\beta}-\sigma}\|w(\cdot, t)\|_{L^{\infty}},
\end{aligned}
$$

where $\bar{\beta} \equiv \min \{\beta, 1 / 2\}$.

Theorem 4 (A Priori Decay Estimate). Let $w$ be defined by (3.1). If $w \in C\left((0, T), L^{1}\right.$ $\left.\cap L^{\infty}\right)$, then

$$
\|w\|_{T} \leqq C
$$

for some constant $C$ independent of $T$, provided $\left\|u_{0}\right\|_{L_{\beta}^{1}}$ is sufficiently small and independent of $T$. 
Once we have (i), (ii), and (iii), then Theorem 2 follows easily: the global existence result follows from a bootstrap procedure using the local existence result and the a priori decay estimation; the convergence rate estimation (1.11) in $L^{1}$ and $L^{\infty}$ follows from (3.2) and (3.3). The estimation (1.11) in $L^{p}$ for general $p, 1 \leqq p \leqq \infty$ can be obtained from interpolation. So, our remaining work is to prove Theorem 4.

Proof of Theorem 4. We expand $w=\sum_{i=1}^{n} w_{i} r_{i}$. Then, from (1.1), (1.5), and (1.8), $w_{i}$ satisfies the following equations:

$$
w_{i t}+\lambda_{i} w_{i x}+S_{i x}+N_{i}(\theta, w)_{x}=w_{i x x}
$$

where

$$
\begin{gathered}
N_{i}(a, b)=l_{i} \cdot N(a, b), \\
N(a, b)=f(a+b)-f^{\prime}(0)(a+b)-\frac{1}{2}\left(f^{\prime \prime}(0) a, a\right), \\
S_{i}= \begin{cases}\frac{1}{2} \sum_{j>r} b_{i j j} \theta_{j}^{2}+\frac{1}{2} \sum_{\substack{j>r \\
j \neq k}}\left(b_{i j k}+b_{i k j}\right) \theta_{j} \theta_{k} & \text { if } i \leqq r \\
\frac{1}{2} \sum_{j \neq i} b_{i j j} \theta_{j}^{2}+\frac{1}{2} \sum_{j \neq k} b_{i j k} \theta_{j} \theta_{k} & \text { if } i>r,\end{cases}
\end{gathered}
$$

and

$$
b_{i j k}=l_{i} \cdot\left(f^{\prime \prime}(0) r_{j}, r_{k}\right) \text {. }
$$

Notice that

$$
N(a, b)=O\left(|a||b|+|a|^{3}+|b|^{2}\right)
$$

for small $a$ and $b$. Also notice that, from (1.4), (1.6), and (1.9), that the initial data $w_{0 i}=w_{i}(\cdot, 0)$ has zero mass:

$$
\int w_{0 i}(x) d x=0, \quad \forall i=1, \ldots, n .
$$

Equation (3.4) is equivalent to the following integral equation:

$$
\begin{aligned}
w_{i}(x, t)= & \int g_{i}(x-y, t) w_{0 i}(y) d y-\int_{0}^{t} \int g_{i}(x-y, t-s) S_{i y}(y, s) d y d s \\
& -\int_{0}^{t} \int g_{i y}(x-y, t-s) N_{i}(\theta(y, s), w(y, s)) d y d s, \\
g_{i}(x, t)= & \frac{1}{\sqrt{4 \pi t}} e^{\frac{\left(x-\lambda_{i} t\right)^{2}}{4 t}} .
\end{aligned}
$$

The strategy is to estimate the right-hand side of (3.8) in terms of $\|w\|_{T}$. It has been shown [1, Lemma 3.1] that the first and the third terms on the right-hand side of (3.8) have the following estimates: Let $\delta=\left\|u_{0}\right\|_{L^{1} \beta}, 0 \leqq \beta \leqq 1$. Then

$$
\begin{gathered}
\left\|g_{i}(\cdot, t) * w_{0 i}\right\|_{T}=O(\delta), \\
\left\|\int_{0}^{t} g_{i y}(\cdot, t-s) * N_{i}(\theta(\cdot, s), w(\cdot, s)) d s\right\|_{T}=O(\delta)\|w\|_{T}+O\left(\delta^{3}\right)+O\left(\|w\|_{T}^{2}\right) .
\end{gathered}
$$


Equation (3.10) is a consequence of (3.6). Equation (3.9) is due to (3.7) and the following important lemma of Kawashima [5, Lemma 8.1].

Lemma 1. Suppose $v_{0} \in L_{\beta}^{1}$ for $0 \leqq \beta \leqq 1$ and $\int v_{0}(x) d x=0$. Then

$$
\left\|D_{x}^{l}\left(g_{i}(\cdot, t) * v_{0}\right)\right\|_{L^{p}}=O(1+t)^{-l / 2-\beta / 2-1 / 2+1 / 2 p}\left\|v_{0}\right\|_{L_{\beta}^{1}}
$$

for $1 \leqq p \leqq \infty$ and $l \geqq 0$.

The estimation of the second term on the right-hand side of (3.8), which we refer as the "convection of the transversal diffusion waves," is not quite the same as before [6, Sect. 7 , or 2 , p. 526]. We give a detailed analysis here.

Among the terms in $S_{i}$, the terms $\theta_{j} \theta_{k}$ with $j \neq k, j>r$ or $k>r$ decay as $e^{-\alpha s}$ for some constant $\alpha>0$, because of (2.3) and $\lambda_{j} \neq \lambda_{k}$. This yields (see [1, Proposition 2.2])

$$
\left\|\int_{0}^{t} g_{i}(\cdot, t-s) *\left(\theta_{j} \theta_{k}\right)_{y} d s\right\|_{T}=O\left(\delta^{2}\right) .
$$

The rest of the terms in $S_{i}$, like $\theta_{j}^{2}$, or $\theta_{j} \theta_{k}$ for $j, k \leqq r$, all have the same expression

$$
\frac{\delta^{2}}{s+1} \phi\left(\frac{y-\lambda_{j}(s+1)}{\sqrt{s+1}}\right)
$$

with $\lambda_{j} \neq \lambda_{i}$ and with some function $\phi$ which has the expression

$$
\phi(\xi)=e^{-\xi^{2} / \alpha} \psi(\xi)
$$

for some positive constant $\alpha$ and for some function $\psi ; \psi$ and all its derivatives are uniformly bounded. Thus, we need to estimate the integral

$$
\int_{0}^{t} \int g_{i}(x-y, t-s) \frac{\delta^{2}}{s+1} \phi\left(\frac{y-\lambda_{j}(s+1)}{\sqrt{s+1}}\right) d y d s,
$$

with $\lambda_{i} \neq \lambda_{j}$. Let us denote this integral by $\xi$. We claim that

$$
\left\|D_{x}^{l} \xi(\cdot, t)\right\|_{L^{p}}=O\left(\delta^{2}\right)(1+t)^{-l / 2-3 / 4+1 / 2 p+\sigma}, \quad 1 \leqq p \leqq \infty,
$$

with $\sigma>0$ being arbitrarily small.

To prove this, we decompose $\xi=\eta+\zeta ; \eta$ satisfies the hyperbolic equation

$$
\begin{gathered}
\eta_{t}+\lambda_{i} \eta_{x}+\left(\frac{\delta^{2}}{t+1} \phi\left(\frac{x-\lambda_{j}(t+1)}{\sqrt{t+1}}\right)\right)_{x}=0, \\
\eta(x, \infty)=0
\end{gathered}
$$

and $\zeta$ satisfies the parabolic equation:

$$
\begin{gathered}
\zeta_{t}+\lambda_{i} \zeta_{x}=\zeta_{x x}+\eta_{x x}, \\
\zeta(x, 0)=-\eta(x, 0) .
\end{gathered}
$$

The function $\eta$ is similar to the hyperbolic wave introduced by Liu; however Eq. (3.15) is much simple (thus simplifying the calculation) and $\eta$ carries zero mass because Eq. (3.15) is in conservation form. We shall prove that $\eta$ has the following pointwise estimate. 
Lemma 2. The solution $\eta$ of (3.15) and (3.16) has the following estimate:

$$
\begin{aligned}
\left|D_{x}^{l} \eta(x, t)\right|= & O\left(\delta^{2}\right) d(x, t)^{-l / 2-3 / 2} \\
& +O\left(\delta^{2}\right)(t+1)^{-l / 2} d(x, t)^{-1} e^{-\gamma\left(x-\lambda_{j}(t+1)\right)^{2} /(t+1)},
\end{aligned}
$$

where

$$
d(x, y)=\left|x-\lambda_{i}(t+1)\right|+\left|\lambda_{i}-\lambda_{j}\right|(t+1),
$$

and $\gamma$ is some positive constant.

A consequence of this lemma is

$$
\left\|D_{x}^{l} \eta(\cdot, t)\right\|_{L^{p}}=O\left(\delta^{2}\right)(1+t)^{-l / 2-1+1 / 2 p} .
$$

The function $\zeta$, by (3.17) and (3.18), satisfies the following integral equation:

$$
\begin{aligned}
\zeta(\cdot, t)= & -g_{i}(\cdot, t) * \eta(\cdot, 0) \\
& -\int_{0}^{\infty} g_{i_{y}}(\cdot, t-s) * \eta_{y}(\cdot, s) d s .
\end{aligned}
$$

From (3.15), (3.16), and (3.19), we obtain $\int \eta(x, 0) d x=0$ and $\eta(\cdot, 0) \in L_{1 / 2-\sigma}^{1}$ for any $\sigma>0$ being arbitrarily small. Thus, Lemma 1 implies

$$
\left\|g_{i}(\cdot, t) * \eta(\cdot, 0)\right\|_{L^{p}}=O\left(\delta^{2}\right)(1+t)^{-3 / 4+1 / 2 p+\sigma} .
$$

The second term on the right-hand side of (3.21) has the following estimate from (3.20),

$$
\begin{aligned}
& \left\|\int_{0}^{t} g_{i_{y}}(\cdot, t-s) * \eta_{y}(\cdot, s) d s\right\|_{L^{p}} \\
& \leqq \int_{0}^{t / 2}\left\|g_{i y y}(\cdot, t-s)\right\|_{L^{p}}\|\eta(\cdot, s)\|_{L^{1}} d s \\
& \quad+\int_{t / 2}^{t}\left\|g_{i_{y}}(\cdot, t-s)\right\|_{L^{1}}\left\|\eta_{y}(\cdot, s)\right\|_{L^{p}} d s \\
& \leqq O\left(\delta^{2}\right) \int_{0}^{t / 2}(t-s)^{-3 / 2+1 / 2 p}(1+s)^{-1 / 2} d s \\
& \quad+O\left(\delta^{2}\right) \int_{t / 2}^{t}(t-s)^{-1 / 2}(1+s)^{-3 / 2+1 / 2 p} d s \\
& \leqq O\left(\delta^{2}\right)(1+t)^{-1+1 / 2 p} .
\end{aligned}
$$

Hence, (3.21), (3.22), and (3.23) yield

$$
\|\zeta(\cdot, t)\|_{L^{p}}=O\left(\delta^{2}\right)(1+t)^{-3 / 4+1 / 2 p+\sigma} .
$$

With (3.20) and (3.24), we complete the proof of claim (3.14) for $\xi$ with $l=0$. Higherorder derivatives of $\xi$ can be estimated in a similar way.

To complete the proof of the theorem, we obtain from (3.9), (3.10), and (3.14) that

$$
\|w\|_{T}=O(\delta)+O(\delta)\|w\|_{T}+O\left(\|w\|_{T}^{2}\right) \leqq C\left(\delta+\delta\|w\|_{T}+\|w\|_{T}^{2}\right)
$$

for some constant $C>0$ independent of $T$. Therefore, $\|w\|_{T}$ is uniformly bounded if $\delta$ is sufficiently small. This completes the proof of Theorem 4. 
Our remaining work is to prove Lemma 2.

Proof of Lemma 2. Without loss of generality, we may assume $\lambda_{i}=0, \lambda_{j}=1$, and $\alpha=1$ in (3.15) and (3.12). We integrate (3.15) along the characteristic line $y=x$ from $s=t$ to $s=\infty$ to obtain

$$
\eta(x, t)=\int_{t}^{\infty} \frac{1}{s+1} \frac{\partial}{\partial x} \phi\left(\frac{x-(s+1)}{\sqrt{s+1}}\right) d s .
$$

Below, $C$ and $\gamma$ represent some positive constants. For $(x, t)$ in the region $x \leqq \frac{1}{2}(t+1)$,

$$
e^{-\frac{(x(s+1))^{2}}{s+1}} \leqq C e^{-\gamma(|x|+(s+1))}
$$

for $s \geqq t$. This implies

$$
|\eta(x, t)| \leqq C e^{-\gamma(|x|+t)}, \text { for } \quad x \leqq \frac{1}{2}(t+1) .
$$

Next, from the self-similarity of $\phi$, we obtain

$$
\begin{aligned}
\frac{\partial \phi}{\partial x}\left(\frac{x-(s+1)}{\sqrt{s+1}}\right) & =\frac{1}{\sqrt{s+1}} \phi^{\prime}\left(\frac{x-(s+1)}{\sqrt{s+1}}\right) \\
& =-\frac{2(s+1)}{x+s+1} \frac{\partial \phi}{\partial s}\left(\frac{x-(s+1)}{\sqrt{s+1}}\right) .
\end{aligned}
$$

This change is legitimate in the region $x>\frac{1}{2}(t+1)$ and $s \geqq t$, where $x+s+1 \neq 0$. Hence, in this region,

$$
\begin{aligned}
\eta(x, t) & =\int_{t}^{\infty} \frac{-2}{x+s+1} \frac{\partial \phi}{\partial s} d s \\
& =\frac{2}{x+t+1} \phi\left(\frac{x-(t+1)}{\sqrt{t+1}}\right)-\int_{t}^{\infty} \frac{2 \phi}{(x+s+1)^{2}} d s .
\end{aligned}
$$

We break the last integral into $\int_{t}^{s^{*}}+\int_{s^{*}}^{\infty}$, where $s^{*}=2 x-1$. Then, for $x>\frac{1}{2}(t+1)$,

$$
\int_{s^{*}}^{\infty} \frac{2|\phi|}{(x+s+1)^{2}} d s \leqq C e^{-\gamma(x+t)}
$$

and

$$
\begin{aligned}
\int_{t}^{s^{*}} \frac{2|\phi|}{(x+s+1)^{2}} d s & \leqq C \int_{t}^{s^{*}} \frac{1}{(x+t+1)^{2}} e^{(x-(s+1))^{2} /\left(s^{*}+1\right)} d s \\
& \leqq C(x+t+1)^{-3 / 2} .
\end{aligned}
$$

Then from (3.26), (3.27), (3.28), and (3.29) the lemma follows for the case of $l=0$. The higher-order derivatives of $\eta$ can be estimated in the same way.

Final Remark. The author recently learned that T.-P. Liu just obtained an optimal $L^{p}$ estimate of the convergence rate to the single-mode diffusion waves. His result, which is an improvement of work in $[1,5]$, is reported in T.-P. Liu, Interactions of Nonlinear Hyperbolic Waves, Preprint, Courant Institute, New York University (1990). 


\section{References}

1. Chern, I-L., Liu, T.-P.: Convergence to diffusion waves of solutions for viscous conservation laws. Commun. Math. Phys. 110, 503-517 (1987)

2. Chern, I-L., Liu, T.-P.: Erratum, Convergence to diffusion waves of solutions for viscous conservation laws. Commun. Math. Phys. 120, 525-527 (1987)

3. Hopf, E.: The partial differential equation $u_{t}+u u_{x}=\mu u_{x x}$. Commun. Pure Appl. Math. 3, 201-230 (1950)

4. Kawashima, S.: Systems of a hyperbolic-parabolic composite type, with applications to the equations of magnetohydrodynamics. Doctoral thesis, Kyoto University (1983)

5. Kawashima, S.: Large-time behavior of solutions to hyperbolic-parabolic systems of conservation laws and applications. Proc. R. Soc. Edinburgh 106 A, 169-194 (1987)

6. Liu, T.-P.: Nonlinear stability of shock waves for viscous conservation laws. Mem. Am. Math. Soc. 56, (328) 1-108 (1985)

7. Liu, T.-P.: Pointwise convergence to $N$-waves for solutions of hyperbolic conservation laws. Bull. Inst. Math. Academia Sinica 15, 1-17 (1987)

8. Liu, T.-P., Xin, Z.-P.: Large-time behaviors of solutions of viscous nonstrictly hyperbolic model. New York University (1990)

9. Matzumura, A., Nishida, T.: The initial value problem for the equations of motion of viscous and heat-conductive gases. J. Math. Kyoto Univ. 20, 67-104 (1980)

10. Shaeffer, D.G., Shearer, M.: The classification of $2 \times 2$ systems of nonstrictly hyperbolic conservation laws, with application to oil recovery. Commun. Pure Appl. Math. 40, 141-178 (1987)

11. Temple, B.: Global solution of the Cauchy problem for a class of $2 \times 2$ nonstrictly hyperbolic conservation laws. Adv. Appl. Math. 3, 335-375 (1982)

Communicated by S.-T. Yau 
